\title{
VALIDAÇÃO DE MÉTODOS ANALÍTICOS: ESTRATÉGIA E DISCUSSÃO
}

NATILENE MESQUITA BRITO * OZELITO POSSIDÔNIO DE AMARANTE JUNIOR ** LUCIANA POLESE ** MARIA LÚCIA RIBEIRO ***

\begin{abstract}
Neste trabalho são apresentadas as definições dos parâmetros (seletividade, limites de detecção e quantificação, exatidão, precisão, linearidade, gráfico analítico, sensibilidade e robustez) considerados nos processos de validação de métodos analíticos. A estratégia a ser adotada para a determinação desses parâmetros depende do propósito e da natureza do método. Exemplos são apresentados para a avaliação dos parâmetros no procedimento de validação.
\end{abstract}

PALAVRAS-CHAVE: MÉTODOS ANALÍTICOS-VALIDAÇÃO; CRITÉRIOS DE VALIDAÇÃO.

* $\quad$ Mestre em Química Analítica pelo Instituto de Química, Universidade Estadual Paulista "Júlio de Mesquita Filho" (UNESP), Araraquara, SP (e-mail: natibrit@posgrad.iq.unesp.br).

** Mestre em Química Analítica pela Universidade Federal do Maranhão (UFMA), Departamento de Tecnologia Química, Centro de Ciências Exatas e Tecnologia (CCET), São Luís, MA.

*** Doutora em Química, Instituto de Química, UNESP, Araraquara, SP.

**** Professora, Doutora em Química Orgânica, Instituto de Química, UNESP, Araraquara - SP. 


\section{INTRODUÇÃO}

O desenvolvimento de um método analítico, a adaptação ou implementação de método conhecido, envolve processo de avaliação que estime sua eficiência na rotina do laboratório. Esse processo costuma ser denominado de validação. Várias definições estão descritas na literatura para validação, tratando-se portanto de termo não-específico. Determinado método é considerado validado se suas características estiverem de acordo com os pré-requisitos estabelecidos. Portanto, existe diferença entre a execução de experimentos que determinam os diversos parâmetros (coleta dos dados experimentais) e a validação. Essa deve avaliar a relação entre os resultados experimentais e as questões que o método se propõe a responder. $O$ objetivo da validação consiste em demonstrar que 0 método analítico é adequado para o seu propósito (1). A validação deve ser considerada quando se desenvolve ou efetua adaptações em metodologias já validadas, inclusão de novas técnicas ou uso de diferentes equipamentos.

A literatura dispõe de vários trabalhos que relatam a validação de métodos analíticos (2-11) e definem os critérios que devem ser seguidos durante seu desenvolvimento. Dentre esses, muitos são das áreas biológica (2-4), farmacêutica (5-7) e química (8-11). Tais artigos abordam os critérios de validação de acordo com sua área específica, enfatizando a exatidão, a precisão e os limites de deteç̧ão e quantificação.

Este trabalho apresenta a definição dos parâmetros requeridos para a validação de métodos analíticos, assim como sugere roteiro para execução desse processo.

\section{CRITÉRIOS DE VALIDAÇÃo}

Os parâmetros de validação de métodos analíticos envolvem Especificidade/Seletividade, Função da Resposta (gráfico analítico), Intervalo de Trabalho, Linearidade, Sensibilidade, Exatidão, Precisão (repetitividade, precisão intermediária e reprodutividade), Limite de Detecção (LD), Limite de Quantificação (LQ) e Robustez.

\subsection{ESPECIFICIDADE/SELETIVIDADE}

O termo especificidade, muitas vezes utilizado como sinônimo de seletividade, define a capacidade do método em detectar o analito de interesse na presença de outros componentes da matriz. Já a seletividade refere-se à capacidade de detecção de substâncias (5,12-13).

O processo para demonstrar a especificidade do método depende do seu objetivo. Em diversas técnicas analíticas (como nas análises cromatográficas, por exemplo) esse parâmetro pode ser estabelecido pela comparação do resultado obtido com a combinação de vários fatores. Como substâncias diferentes podem apresentar respostas similares em dadas condições deve-se proceder à análise, seguida por outras técnicas comprobatórias (como cromatografia ou eletroforese acoplada à espectrometria de massas). Outra maneira de avaliar a especificidade envolve a adição de padrão analítico (muito empregada em análises por espectrometria de absorção ou de emissão atômica) ou a comparação com padrão externo (5).

\subsection{FUNÇÃO DA RESPOSTA (GRÁFICO ANALÍTICO)}

O gráfico analítico deve apresentar os dados estatísticos de intersecção, da equação da regressão linear, o coeficiente de correlação ou de determinação e a concentração estimada dos calibradores 
(soluções-padrão). Assim, torna-se necessário o uso de número suficiente de soluções-padrão para definir adequadamente a relação entre a concentração e a resposta. O gráfico analítico pode ser construído usando-se, no mínimo, cinco valores de concentração enquadrados no intervalo definido (5, 8, 13-15).

A suposição clássica do gráfico de calibração é que a resposta instrumental y está linearmente relacionada com a concentração do padrão y, tal como:

$$
y=F(x)+e y
$$

Na qual: ey = erro randômico ou indeterminado (distribuição normal); $y=$ sinal analítico (ou resposta instrumental).

Geralmente, o erro randômico é desprezado na relação, ficando $y=F(x)$ :

$$
F(x)=B+S x
$$

$\mathrm{Na}$ qual: $\mathrm{B}=$ média das medidas do branco (ou linha de base); $\mathrm{S}=$ sensibilidade do método; $\mathrm{x}=$ concentração do analito na amostra.

Julga-se satisfatória a linearidade do gráfico quando o coeficiente de correlação da reta obtida não é estatisticamente diferente da unidade (9). No caso, considera-se:

$\begin{array}{ll}R=1 & \text { Correlação perfeita } \\ 0,91<R<0,99 & \text { Correlação fortíssima } \\ 0,61<R<0,91 & \text { Correlação forte } \\ 0,31<R<0,60 & \text { Correlação média } \\ 0,01<R<0,30 & \text { Correlação fraca } \\ R=\text { zero } & \text { Correlação nula }\end{array}$

Embora sempre se busque obter relação linear entre a propriedade a ser medida e a concentração ou quantidade do analito pode-se também admitir a relação não-linear (16) (por exemplo, nas análises eletroquímicas, utilizando eletrodos de íon seletivo ou biossensores).

\subsection{INTERVALO DE TRABALHO}

O intervalo do método analítico corresponde à faixa do maior ao menor nível que possa ser determinado com precisão e exatidão, usando a linearidade do método (13-15). Geralmente, os analistas seguem o caminho inverso. Primeiro, selecionam o intervalo de trabalho (baseado no nível de concentração do analito que desejam estudar) e depois determinam se a relação sinal versus concentração é linear.

\subsection{LINEARIDADE}

A linearidade refere-se à capacidade do método de gerar resultados linearmente proporcionais à concentração do analito, enquadrados em faixa analítica especificada. Esse parâmetro pode ser demonstrado pelo coeficiente de correlação do gráfico analítico (13-17), que não deve ser estatisticamente 
diferente de 1 (avaliado pelo teste " $t$ " de Student), observando-se que a inclinação da reta seja diferente de zero. Assim, é necessário obter coeficiente de correlação estatisticamente igual a um e coeficiente angular diferente de zero.

A linearidade pode ser avaliada por meio da análise de variância ponderada (ANOVA ponderada). Considera-se que o gráfico analítico satisfaz o critério de linearidade quando quatro gráficos mostrarem resultado positivo para esse teste ou quando três forem lineares $(12,18)$ e aquele que não satisfizer as condições no nível de significância de 0,05 as satisfaça no nível de 0,01 ( $\mathrm{Fa} 0,05<\mathrm{Fobtido<Fa} \mathrm{0,01).} \mathrm{Outra}$ forma de avaliar a linearidade é por meio da relação entre a concentração recuperada e a de fortificação, que deve ser linear (2). Assim, estima-se a capacidade do método em desviar-se linearmente da recuperação ideal (100\%) (16).

\subsection{SENSIBILIDADE}

A sensibilidade é a capacidade do método em distinguir, com determinado nível de confiança, duas concentrações próximas (16). Sob o ponto de vista prático, a sensibilidade constitui o coeficiente angular do gráfico analítico $(3,19)$ expresso como:

$$
S=\frac{d \gamma}{d \chi}
$$

Em métodos sensíveis, uma pequena diferença na concentração do analito causa grande variação no valor do sinal analítico medido. Esse critério expressa a capacidade do procedimento analítico gerar variação no valor da propriedade monitorada ou medida, causada por pequeno incremento na concentração ou quantidade do analito. Entretanto, tornou-se comum o uso errôneo desse termo para designar método com baixo limite de detecção (LD) (16).

\subsection{EXATIDÃO}

A exatidão, definida como a concordância entre o valor real do analito na amostra e o estimado pelo processo analítico $(7,13-16,20-22)$, constitui a chave para o propósito da validação. Os quatro métodos principais, propostos para o estudo da exatidão, são baseados no uso de material de referência certificado (MRC), na comparação do método proposto com um método de referência, no uso de ensaios de recuperação na matriz e em estudos colaborativos (20). Os MRC (quando disponíveis) são os materiais de controle preferidos, pois estão diretamente relacionados com padrões internacionais. O processo de avaliação por meio de MRC consiste em analisar número suficiente de replicatas desse material e comparar os resultados obtidos com o valor certificado. Entretanto, o alto custo do MRC e a abrangência limitada de matrizes e analitos restringem seu uso (21).

A exatidão também pode ser estabelecida mediante comparação entre os valores obtidos pelo método proposto com os valores obtidos para as mesmas amostras com outro método validado (método com precisão e exatidão avaliadas). Após análise de diferentes amostras com ambos os métodos, as diferenças obtidas para cada amostra são calculadas e comparadas com o valor desejado (nesse caso, zero). Estabelece-se, então, o nível de confiança de acordo com o intervalo de concentração (menores valores de concentração causam maior dispersão dos dados aumentando o limite de confiança) (16). Entretanto, nem sempre se encontra método de referência preexistente, impossibilitando a utilização desse tipo de proposta (20).

Estudos colaborativos implicam na aceitação de pelo menos oito laboratórios (número mínimo) em desenvolver determinado método. Somente quando for impossível reunir tal número de laboratórios, o estudo poderá ser conduzido com o mínimo absoluto de cinco participantes (22). A maior dificuldade 
freqüentemente encontrada quando se tenta estabelecer a exatidão do método por meio de estudos colaborativos envolve a garantia de estabilidade do analito (assegurar que a concentração do analito, a ser determinada na amostra, permaneça estável durante o decorrer do estudo). Isso torna-se particularmente difícil com compostos lábeis $(7,21)$.

No processo de validação em estudos colaborativos usa-se a análise de variância (ANOVA), que determina a existência de diferenças significativas entre os valores obtidos pelos laboratórios envolvidos (16).

O ensaio de recuperação constitui o método mais utilizado para validação de processos analíticos. A recuperação está relacionada com a exatidão, pois reflete a quantidade de determinado analito, recuperado no processo, em relação à quantidade real presente na amostra. A exatidão é expressa como erro sistemático percentual, inerente ao processo $(12,20)$. O erro sistemático ocorre pela perda da substância devido à baixa recuperação da extração, medidas volumétricas imprecisas ou substâncias interferentes na amostra (entre outros).

O estudo da recuperação consiste na "fortificação" da amostra (Figura 1), ou seja, na adição de soluções com diferentes concentrações do analito de interesse seguida pela determinação da concentração do analito adicionado. Calcula-se a quantidade percentual recuperada pelo processo (3) usando a fórmula:

$$
\operatorname{Re} c \%=\frac{\lfloor\text { valor obtido }- \text { valor real }\rfloor}{\text { valor real }} \times 100
$$

\section{FIGURA 1 - PROCESSO DE FORTIFICAÇÃO DAS AMOSTRAS}

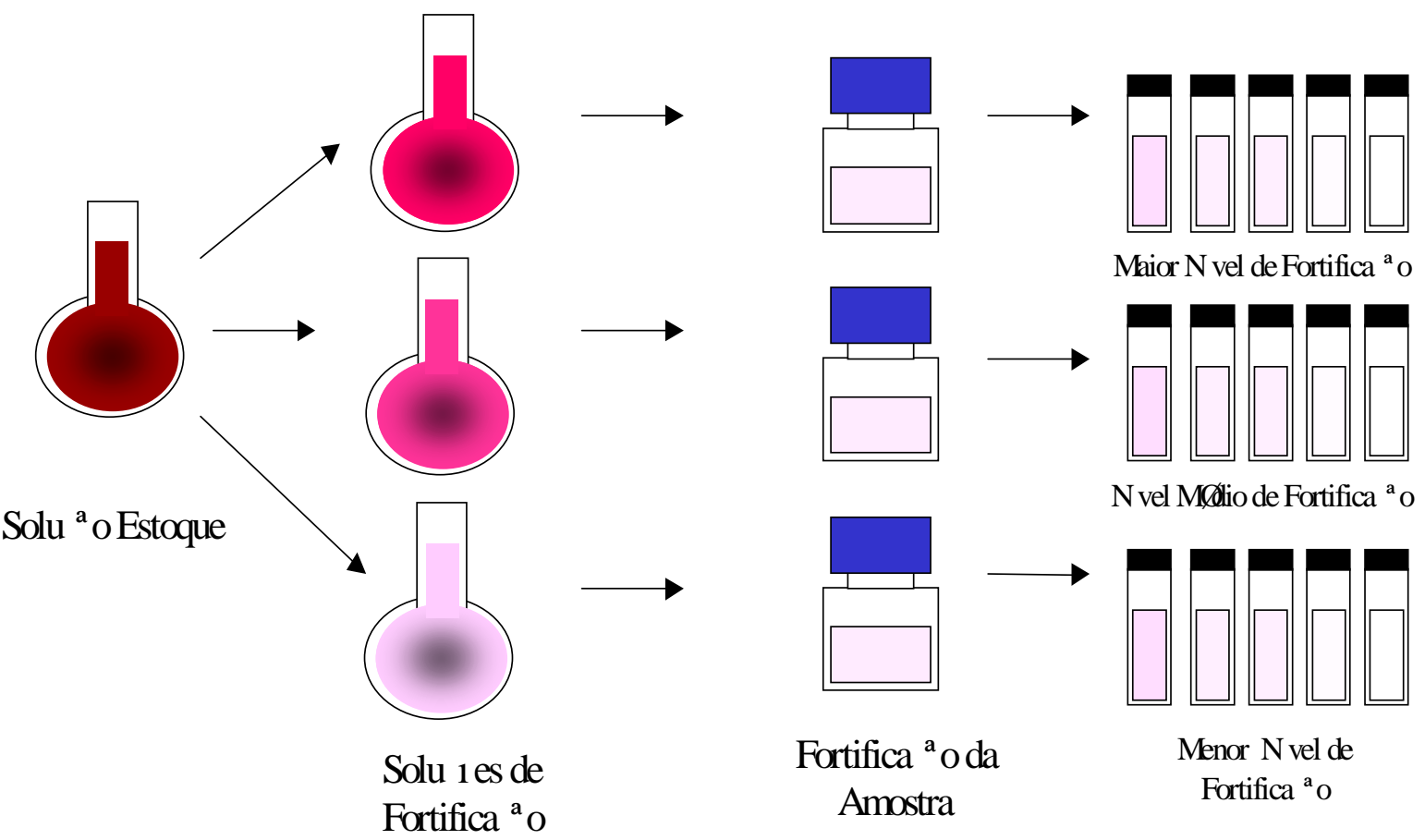

FONTE: BRITO, 2001 (13). 
No estudo da recuperação são realizados testes de significância, utilizando o teste "t" de Student de acordo com a seguinte fórmula (23):

$$
t=\frac{(\operatorname{Re} c-100)}{\frac{S_{\operatorname{Re} c}}{\sqrt{n-1}}}
$$

Na qual: $R e c=$ a média das recuperações obtidas para $n$ repetições; $100=$ a recuperação percentual desejada; $\mathrm{n}=0$ número de determinações (trabalha-se com no mínimo 5 repetições); $S R e c=0$ desviopadrão das recuperações.

Se o valor de t obtido estiver enquadrado no intervalo estabelecido pelo valor tabelado, para $\mathrm{n}$ 1 graus de liberdade em dado nível de significância, então o método será considerado exato (24). Além de efetuar o teste "t" de Student, existem valores críticos aceitáveis de acordo com a concentração do analito em estudo. Esses valores são estimados considerando-se que análises de elementos majoritários costumam apresentar erros sistemáticos relativos muito inferiores àqueles obtidos para analitos em concentrações muito pequenas. Tais valores, sugeridos pelo manual da Association of Official Analytical Chemists (AOAC) são apresentados na Tabela 1.

\section{TABELA 1 - RECUPERAÇÃO DO ANALITO EM FUNÇÃO DA CONCENTRAÇÃO}

\begin{tabular}{l|c}
\hline Concentração do analito (\%) & Intervalo de Recuperação Aceito (\%) \\
\hline$\geq 10$ & $98-102$ \\
$\geq 1$ & $97-103$ \\
$\geq 0,1$ & $95-105$ \\
$\geq 0,01$ & $90-107$ \\
$\geq 0,001-\geq 0,00001$ & $80-110$ \\
$\geq 0,000001$ & $60-115$ \\
$\geq 0,0000001$ & $40-120$ \\
\hline
\end{tabular}

Fonte: BRITO, 2001 (13).

Deve-se ressaltar que o processo de fortificação tenta simular condições reais, não interferindo significativamente nas interações que ocorrem na própria amostra. Isso nem sempre é possível, visto que a solução de fortificação é preparada com solventes orgânicos, geralmente não-presentes na matriz. Por essa razão, tenta-se usar volume reduzido de solução de fortificação e condições que alterem a amostra o mínimo possível $(16,25)$.

\subsection{PRECISÃO}

O parâmetro que avalia a proximidade entre várias medidas efetuadas na mesma amostra é a precisão do processo analítico. Usualmente, é expressa como o desvio-padrão, variância ou coeficiente de variação (CV) de diversas medidas $(2,17)$. O CV é dado pela seguinte equação: 


$$
C V \%=\frac{s}{M} \times 100
$$

Na qual: $\mathbf{s}=$ desvio-padrão das recuperações e $\mathrm{M}=$ média das recuperações.

Para análise de resíduos (26) de pesticidas, por exemplo, consideram-se aceitáveis coeficientes de variação de até $20 \%$. Segundo WOOD (27), o coeficiente de variação (ou desvio-padrão relativo) está relacionado com o nível de concentração do analito na amostra, definido pela equação: $\mathrm{CV}(\%)=$ $2^{(1-0,5 \log C)}$, na qual $C$ é a concentração do analito. Desse modo, substituindo-se os níveis de concentração nessa equação obtém-se os valores CV apresentados na Tabela 2.

\section{TABELA 2 - COEFICIENTES DE VARIAÇÃO EM FUNÇÃO DO NÍVEL DE CONCENTRAÇÃO DO} ANALITO

\begin{tabular}{l|c}
\hline $\begin{array}{l}\text { Concentração do analito } \\
(\%)\end{array}$ & Coeficiente de Variação (\%) \\
\hline $1(100 \%)$ & 2,8 \\
$10^{-1}$ & 4 \\
$10^{-2}(1 \%)$ & 5,6 \\
$10^{-3}$ & 8 \\
$10^{-4}$ & 11 \\
$10^{-5}$ & 16 \\
$10^{-6}(\mathrm{ppm})$ & 23 \\
$10^{-7}$ & 32 \\
$10^{-8}$ & 45 \\
$10^{-9} \quad(\mathrm{ppb})$ & \\
\hline
\end{tabular}

Fonte: WOOD, 1999 (27).

A precisão pode ser considerada no nível de repetitividade, de precisão intermediária e de reprodutividade $(2,3,13-15,22)$. A repetitividade expressa a precisão nas mesmas condições de operação (equipamento, analista, reagentes, dia e mesmas condições ambientais) em pequeno espaço de tempo. Repetitividade, também conhecida como precisão intra-ensaios (13-15), pode ser avaliada com no mínimo nove determinações dentro do intervalo de três diferentes concentrações e três replicatas cada, ou com no mínimo seis determinações para uma única concentração-teste (14). A precisão intermediária expressa as variações no mesmo laboratório que envolvem diferentes dias, diferentes analistas e diferentes equipamentos, entre outros $(13,14)$. Tal termo tem sido pouco empregado e esses testes não são comumente citados na literatura. A reprodutividade expressa a precisão entre laboratórios, mediante estudos colaborativos usualmente aplicados para padronização de metodologias $(14,15)$. O método será considerado preciso quando apresentar níveis de variações dentro do aceitável para cada nível de concentração conforme apresentado na Tabela 2. 


\subsection{LIMITE DE DETECÇÃO}

A menor concentração do analito que pode ser detectada, mas não necessariamente quantificada, sob condições experimentais estabelecidas constitui o limite de detecção (13-15, 18, 28-31).

Analistas, quando desenvolvem métodos novos ou modificados para análise de traços, freqüentemente definem Limites de Detecção (LD) em termos do desvio-padrão de medidas do branco. Entretanto, sendo o branco constituído de todos os reagentes, com exceção da amostra e do analito, não reproduz as mesmas condições a que o analito estará sujeito durante a análise $(16,25,28)$. O LD determinado dessa forma pode avaliar mudanças ocorridas durante o desenvolvimento do método, ou para comparação de instrumentos. Entretanto, em relação à análise prática, esses valores são extremamente baixos e não representam a realidade $(16,25,28)$. O limite de detecção pode ser determinado mediante o sinal/ruído, o desvio-padrão da resposta e do coeficiente angular e por processos estatísticos.

O sinal/ruído pode ser aplicado somente para processos analíticos que exibem linha de base. A determinação da razão sinal/ruído é realizada por meio da comparação dos sinais medidos da amostra com baixas concentrações conhecidas do analito com as do branco, estabelecendo-se a concentração mínima na qual o analito pode ser detectado. A razão sinal/ruído com valor 3 é geralmente considerada aceitável para estimar o limite de detecção (13-15, 31).

O limite de detecção pode ser expresso pela equação abaixo (13-15, 28-31):

$$
L D=\frac{3 \times s}{S}
$$

Na qual: $\mathbf{S}=$ desvio-padrão da resposta; $S$ = o coeficiente angular do gráfico de calibração (sensibilidade do aparelho).

No caso dos processos estatísticos, utiliza-se o "teste de hipótese" para estimar o LD com valores obtidos de várias medidas do branco pela seguinte equação $(16,25,26)$ :

$$
L D=\frac{2 \times t_{n, 95 \%} \times s_{B}}{S}
$$

Na qual: $\mathrm{t}_{n, 95 \%}=$ valor tabelado em função de $\mathrm{n}$ (número de análises); $s_{B}=$ desvio-padrão do branco; $\mathrm{S}=$ sensibilidade do aparelho.

Quando se obtém poucos valores de medidas do branco ou quando tais valores se apresentam como linha de base, esse tipo de estimativa pode não ser adequado.

OLD pode ser estimado pelos experimentos de recuperação (26) mediante o desvio-padrão $\left(\mathrm{s}_{A}\right)$ do menor nível de fortificação, calculando-se $\mathrm{s}_{\mathrm{com}}$ a partir de $\mathrm{s}_{A}$ e $\mathrm{s}_{B}$ :

$$
s_{c o m}=\sqrt{\frac{(m-1) s_{A}{ }^{2}+(n-1) s_{B}{ }^{2}}{m+n-2}}
$$

Na qual: $\mathrm{m}=$ o número de determinações do menor nível de fortificação; $\mathrm{n}=$ o número de determinações do branco; $\mathrm{S}_{A}=$ desvio-padrão da amostra do menor nível de fortificação; $\mathrm{S}_{B}=$ desvio-padrão do branco. A partir desse valor estima-se o limite de detecção pela equação (26):

$$
L D=\frac{2 \times t_{n, 95 \%} \times s_{\text {com }}}{S}
$$


No processo estatístico são considerados todos os efeitos a que o método está sujeito (variações do branco e variações da amostra fortificada no menor nível).

\subsection{LIMITE DE QUANTIFICAÇÃO}

O limite de quantificação é definido como a menor concentração do analito, que pode ser quantificada na amostra, com exatidão e precisão aceitáveis, sob as condições experimentais adotadas $(13-15,18,28-31)$. Pode ser estimado por meio do sinal/ruído, do desvio-padrão e por processos estatísticos.

O procedimento sinal/ruído pode ser aplicado somente para processos analíticos que exibem linha de base. Efetua-se a determinação da razão sinal/ruído por meio da comparação dos sinais medidos da amostra com baixas concentrações conhecidas do analito com as do branco, estabelecendo-se a concentração mínima na qual o analito pode ser quantificado (13-15, 31). Exemplo de razão sinal/ruído típica é 10:1.

O limite de quantificação (LQ) pode ser expresso pela equação abaixo $(13-15,18,28-31)$ :

$$
L Q=\frac{10 \times s}{S}
$$

Na qual: $\mathrm{S}$ = desvio-padrão da resposta; $\mathrm{S}=0$ coeficiente angular do gráfico de calibração (sensibilidade do método).

De acordo com o procedimento estatístico utilizado para análise de resíduos de pesticidas, o LQ corresponde ao menor nível de fortificação estudado $(16,25,26)$. A recuperação pode variar entre 70 e $120 \%$, com coeficiente de variação de até $20 \%$.

\subsection{ROBUSTEZ}

Segundo a International Conference on Harmonisation $(\mathrm{ICH})$, a robustez do método é a medida da sua capacidade de permanecer inalterado sob pequenas, mas estudadas variações nos parâmetros do método e prover indicação da sua dependência durante o uso normal $(24,32,33)$.

A avaliação da robustez pode ser considerada durante a fase de desenvolvimento e depende do tipo de processo em estudo. Nos testes de robustez são aplicados experimentos estatísticos que examinam, simultaneamente, os efeitos de alterações em diferentes variáveis do método. No caso de métodos cromatográficos, as variações referem-se a diferentes tipos de colunas, temperatura e fluxo, entre outras (32).

Os testes de robustez, em geral, servem para indicar os fatores que podem influenciar, significantemente, a resposta do método estudado. Tal fato fornece a dimensão do problema que ocorre quando o método é repetido em diferentes condições ou é transferido, por exemplo, para outro laboratório (33).

\section{ESTRATÉGIA DE VALIDAÇÃO EM FUNÇÃO DA NATUREZA E DO PROPÓSITO DO MÉTODO}

A estratégia a ser seguida na validação do método analítico apresenta natureza cíclica, semelhante a uma corrente de decisões, ações e procedimentos (34), como mostrado esquematicamente na Figura 2. 
Caso o último passo da validação (interpretação dos resultados da análise) não forneça resposta satisfatória, o ciclo da análise poderá ser refeito depois da adaptação de um ou mais passos.

Para que os resultados sejam confiáveis é imperioso que as variações analíticas sejam mantidas dentro de limites preestabelecidos por critérios baseados na finalidade de análise, sistemas analíticos e estudos estatísticos (12).

Os critérios a serem aplicados para validação de determinado método depende da sua natureza e propósito. Usa-se a distinção entre método "novo", "padrão" e "modificado" para caraterizar sua natureza. Método padrão, nessa definição, é o método completamente validado (com todos os parâmetros estabelecidos). Por outro lado, os métodos são também classificados de acordo com o seu propósito, por exemplo, qualitativo/quantitativo ou análise traços versus componente majoritário (34). A validação requerida para tais métodos está resumida na Figura 3.

\section{FIGURA 2 - ANÁLISE QUÍMICA COMO UM PROCESSO CÍCLICO}

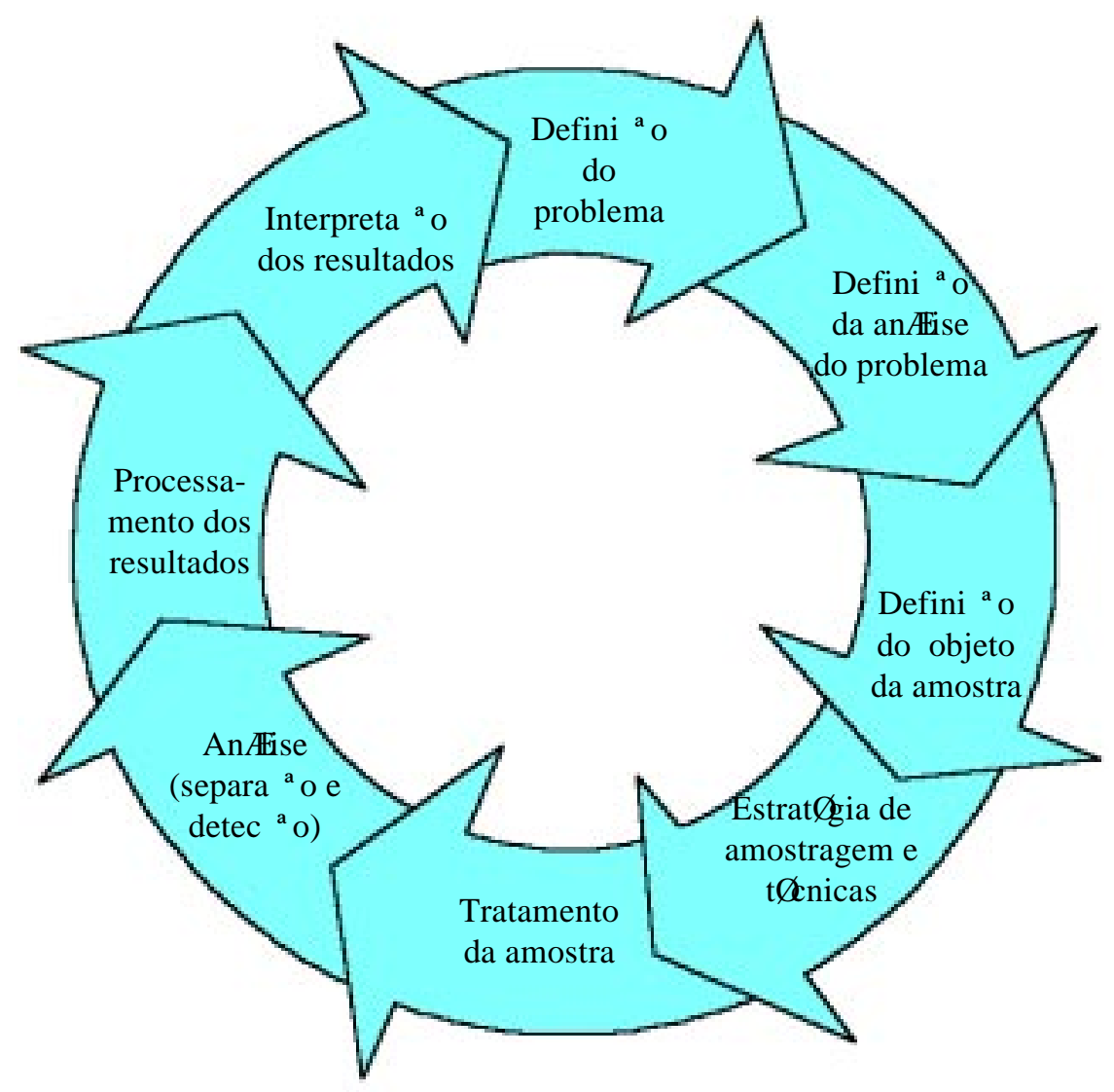

Fonte: Brito, 2001 (13).

Os parâmetros exatidão e precisão constituem a chave para o processo de validação. São requeridos para todos os estudos de validação de métodos, exceto para aqueles com propósito qualitativo, que se destinam apenas a acusar a presença de um analito. Para a análise quantitativa de elementos traços é necessário validar os limites de detecção e de quantificação. Já para análises qualitativas é obrigatório validar apenas o limite de detecção. A avaliação do parâmetro da linearidade é obrigatória para métodos novos e análises qualitativas de componentes majoritários. 

EM ESTUDO
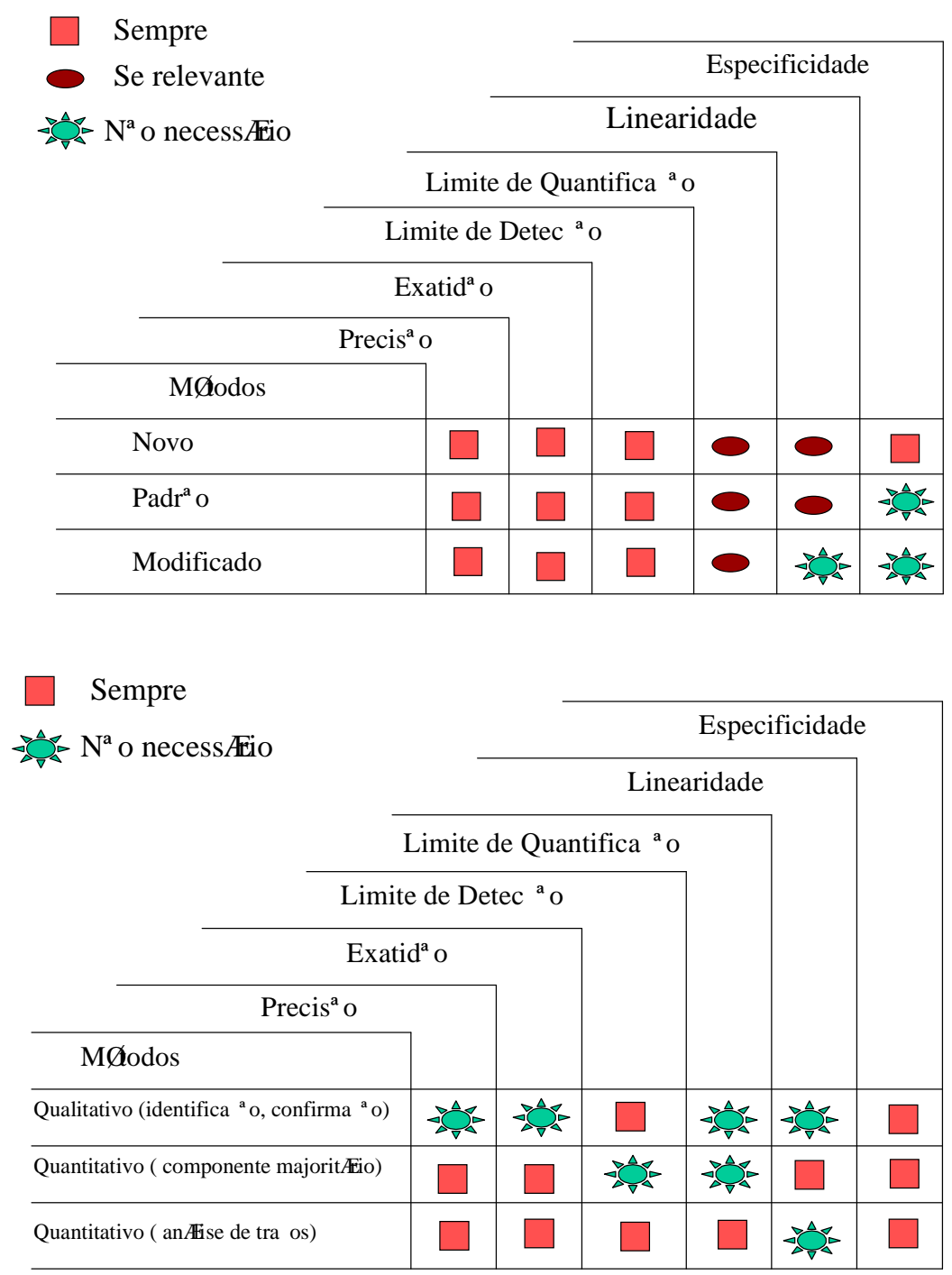

Fonte: Brito, 2001 (13).

\section{ESTUDO DE CASO: ANÁLISE DE HERBICIDAS EM AMOSTRAS DE SOLO}

Para avaliação dos parâmetros tratados, o trabalho de POLESE et al. sobre análise de resíduos de pesticidas foi selecionado na literatura (35) e submetido aos critérios aplicáveis.

\subsection{FUNÇÃO DA RESPOSTA (GRÁFICO ANALÍTICO), INTERVALO DE TRABALHO E SENSIBILIDADE}

No estudo escolhido não foi investigada a linearidade do método, que seria a busca pelo intervalo de concentrações para obter relação linear entre a concentração e o sinal de resposta (altura do pico). Os autores selecionaram a faixa de concentração de trabalho indicada aos níveis de concentração que almejavam investigar e verificaram se este intervalo apresentava a relação linear desejada. Nesse procedimento, o intervalo de trabalho foi definido pelo analista em função da resposta (ou gráfico analítico) para obter a sensibilidade do método (Tabela 3). 
Observou-se que o intervalo de trabalho escolhido guarda excelente relação linear com o sinal analítico, denotado pelos valores de coeficiente de correlação extremamente próximos de 1 . Do mesmo modo, o método apresentou grande sensibilidade, pois os coeficientes angulares das retas apresentaram valores elevados. Isso significa que com pequenas variações na concentração obtêm-se grandes variações nos sinais medidos, garantindo a diferenciação entre duas concentrações bastante próximas.

TABELA 3 - EQUAÇÕES DO GRÁFICO ANALÍTICO, COEFICIENTES DE CORRELAÇÃO E INTERVALO DE TRABALHO

\begin{tabular}{c|c|c|c}
\hline Pesticidas & $\begin{array}{c}\text { Intervalo de } \\
\text { trabalho } \\
(\mathbf{m} \mathbf{g} / \mathbf{L})\end{array}$ & Equa I es & $\begin{array}{c}\text { Coeficientes } \\
\text { de } \\
\text { correla a o }\end{array}$ \\
\hline Diisopropilatrazina & 0,2 a 5,2 & $y=4355,7 x-839,83$ & 0,9992 \\
Dietilatrazina & 0,2 a 4,7 & $y=5496 x-804,23$ & 0,9995 \\
Trifluralina & 0,5 a 10,2 & $y=1742,5 x-322,52$ & 0,9999 \\
Simazina & 0,2 a 5,0 & $y=7829,9 x-1189,2$ & 0,9994 \\
Atrazina & 0,3 a 5,3 & $y=6671 x-804,78$ & 0,9997 \\
Metribuzina & 0,3 a 5,3 & $y=2852,7 x-234,72$ & 1,0000 \\
Metolaclor & 0,5 a 10,0 & $y=1358,4 x-24,886$ & 0,9998 \\
\hline
\end{tabular}

\subsection{EXATIDÃO E PRECISÃO}

O cálculo da recuperação para amostras fortificadas pode ser realizado computando-se as recuperações individuais para cada nível de fortificação do analito(i), avaliando-se a recuperação por meio de sua média (ii), ou analisando a regressão linear da concentração obtida e a concentração de fortificação (iii) (21). Para ilustrar as diferentes formas de avaliação foram utilizados os resultados de POLESE et al. (35) referentes à análise de sete herbicidas por Cromatografia em Fase Gasosa com Detector Termiônico Específico (CG-DTE) em amostras de solo.

\subsection{AVALIAÇÃO DA EXATIDÃO PELO ESTUDO DAS RECUPERAÇÕES INDIVIDUAIS}

Para avaliar a abrangência da exatidão foram estudados três níveis de fortificação e, para cada nível, efetuadas quatro determinações (35). Os valores de recuperação obtidos para os pesticidas são apresentados na Tabela 4.

Usou-se o teste de hipótese para confirmar a exatidão do método, sendo estabelecida como hipótese nula $\left(\mathrm{H}_{0}\right)$ : Rec. $=100 \%$ e como hipótese alternativa $\left(\mathrm{H}_{1}\right)$ : Rec. $\neq 100 \%$ (teste bilateral). $\mathrm{O}$ teste $t$ aplicado, com $95 \%$ de confiança e $n-1$ graus de liberdade, apresentou valores calculados entre os intervalos estabelecidos pelo valor tabelado $(4,17)$, com exceção dos resultados obtidos para Diisopropilatrazina (1,0 e 2,0 mg kg$\left.{ }^{-1}\right)$, Trifluralina $\left(4,0 \mathrm{mg} \mathrm{kg}^{-1}\right)$, Simazina $\left(2,0 \mathrm{mg} \mathrm{kg}^{-1}\right)$, Atrazina (2,0 $\left.\mathrm{mg} \mathrm{kg}^{-1}\right)$, Metribuzina ( 1,0 e 2,0 $\mathrm{mg} \mathrm{kg}^{-1}$ ) e Metolaclor $\left(4,0 \mathrm{mg} \mathrm{kg}^{-1}\right)$. Para estes compostos, nos níveis de fortificação citados, pode-se afirmar que existem diferenças significativas entre as recuperações obtidas e o valor esperado (100\%), com 95\% de confiança e 3 graus de liberdade. 
TABELA 4 - RECUPERAÇÕES PERCENTUAIS E CV DOS COMPOSTOS ANALISADOS POR CG

\begin{tabular}{|c|c|c|c|c|}
\hline Pesticidas & $\begin{array}{c}\text { N vel } \\
\mathrm{mg} \mathrm{kg}^{-1}\end{array}$ & $\begin{array}{c}\text { Recupera }{ }^{a} 0 \\
\text { MØdia (\%) }\end{array}$ & CV \% & $t_{\text {calculado }}$ \\
\hline \multirow{3}{*}{ Diisopropilatrazina } & 0,1 & 84 & 12,0 & $-2,75$ \\
\hline & 1,0 & 81 & 3,6 & $-11,29$ \\
\hline & 2,0 & 83 & 3,6 & $-9,85$ \\
\hline \multirow{3}{*}{ Dietilatrazina } & 0,1 & 95 & 8,4 & $-1,08$ \\
\hline & 1,0 & 103 & 11,0 & 0,46 \\
\hline & 2,0 & 92 & 6,8 & $-2,21$ \\
\hline \multirow{3}{*}{ Trifluralina } & 0,2 & 109 & 10,5 & 1,36 \\
\hline & 2,0 & 103 & 8,0 & 0,63 \\
\hline & 4,0 & 115 & 3,6 & 6,27 \\
\hline \multirow{3}{*}{ Simazina } & 0,1 & 93 & 8,0 & $-1,63$ \\
\hline & 1,0 & 102 & 5,8 & 0,58 \\
\hline & 2,0 & 95 & 2,0 & 4,56 \\
\hline \multirow{3}{*}{ Atrazina } & 0,1 & 105 & 5,5 & 1,50 \\
\hline & 1,0 & 102 & 3,0 & 1,13 \\
\hline & 2,0 & 108 & 1,2 & 10,69 \\
\hline \multirow{3}{*}{ Metribuzina } & 0,1 & 98 & 12,7 & $-0,28$ \\
\hline & 1,0 & 109 & 3,8 & 3,76 \\
\hline & 2,0 & 113 & 1,3 & 15,32 \\
\hline \multirow{3}{*}{ Metolaclor } & 0,2 & 104 & 2,1 & 3,17 \\
\hline & 2,0 & 95 & 5,3 & $-1,72$ \\
\hline & 4,0 & 112 & 2,6 & 7,14 \\
\hline
\end{tabular}

$\mathrm{n}=4 ; \mathrm{CV}=$ Coeficiente de Variação. Fonte: POLESE et al., 2002 (35).

Para o teste t com $99 \%$ de confiança (teste menos restritivo) tem-se o valor tabelado de $t$ igual a 7,45. Os compostos Diisopropilatrazina (1,0 e 2,0 mg kg-1), Atrazina $\left(2,0 \mathrm{mg} \mathrm{kg}^{-1}\right)$ e Metribuzina $\left(1,0\right.$ e $\left.2,0 \mathrm{mg} \mathrm{kg}^{-1}\right)$ apresentaram diferenças significativas entre os valores obtidos e o esperado (100\%). Embora não satisfaça esse teste, o método foi considerando suficientemente exato, pois as recuperações estão compreendidas na faixa entre 70 e $120 \%$, estabelecida pela literatura na área de análise de resíduos de pesticidas $(13,25,26,35)$. Apesar dos valores de recuperação encontrados serem significativamente diferentes de $100 \%$ são aceitáveis por se repetirem em todas as amostras, evidenciando a consistência do método. Fato comprovado pelo estudo da precisão.

Para o estudo da precisão, os coeficientes de variação (obtidos a partir dos valores referentes ao ensaio de recuperação) foram considerados adequados (iguais ou inferiores a $20 \%$ ) conforme recomendado por THIER e ZEUMER $(13,25,26,35)$. Como todos os valores de CV mostraram-se inferiores ao estabelecido, o método proposto foi considerado preciso.

\subsection{AVALIAÇÃO DA EXATIDÃO PELO ESTUDO DAS MÉDIAS DAS RECUPERAÇÕES}

Para avaliar a exatidão pode-se calcular as médias dos três níveis de fortificação. Os valores calculados para os resultados obtidos por POLESE et al. (35), apresentados na Tabela 5, permaneceram enquadrados na faixa estabelecida para a análise de resíduos de pesticidas (entre 70 e 120\%) descritos na literatura $(13,25,26,35)$.

Os valores foram submetidos ao teste de hipótese, sendo estabelecida como hipótese nula $\left(H_{0}\right)$ : Rec. $=100 \%$ e como hipótese alternativa $\left(H_{1}\right)$ : Rec. $\neq 100 \%$ (teste bilateral). Obteve-se a aceitação da hipótese nula para todos os compostos com exceção dos valores observados para Diisopropilatrazina, tanto com $95 \%$ quanto com $99 \%$ de confiança. Os valores de CV indicaram que o método mostrou-se suficientemente preciso de acordo com o estabelecido na literatura $(13,25,26$, 35). Cabe ressaltar que esse método de avaliação fornece visão muito geral dos valores de recuperação obtidos, deixando de esclarecer quais níveis de fortificação não se enquadrarão no intervalo de linearidade do método (ao contrário do procedimento anterior). 
TABELA 5 - MÉDIAS DAS RECUPERAÇÕES E CV DOS COMPOSTOS ANALISADOS POR CG

\begin{tabular}{c|c|c|c|c}
\hline Pesticidas & $\begin{array}{c}\mathbf{N} \text { vel } \\
\mathbf{m ~ g ~ k g}^{-1}\end{array}$ & $\begin{array}{c}\text { Recupera a o } \\
\text { MØdia (\%) }\end{array}$ & $\mathbf{C V} \%$ & $\mathbf{t}_{\text {calculado }}$ \\
\hline Diisopropilatrazina & $0,1-2,0$ & 83 & 1,8 & $-16,05$ \\
\hline Dietilatrazina & $0,1-2,0$ & 97 & 5,9 & $-0,83$ \\
\hline Trifluralina & $0,2-4,0$ & 109 & 5,5 & 2,12 \\
\hline Simazina & $0,1-2,0$ & 97 & 4,9 & $-1,00$ \\
\hline Atrazina & $0,1-2,0$ & 105 & 2,9 & 2,36 \\
\hline Metribuzina & $0,1-2,0$ & 107 & 7,3 & 1,21 \\
\hline Metolaclor & $0,2-4,0$ & 104 & 8,2 & 0,61 \\
\hline
\end{tabular}

$\mathrm{n}=4 ; \mathrm{CV}=$ Coeficiente de Variação.

Fonte: POLESE et al., 2002 (35).

\subsection{AVALIAÇÃO DA EXATIDÃO PELO ESTUDO DA REGRESSÃO LINEAR}

Pelo estudo da regressão linear pode-se verificar se as recuperações apresentam distribuição linear com relação aos níveis de fortificação (os coeficientes de correlação fornecem a informação relativa a esse aumento proporcional). Cabe ressaltar que os parâmetros mais importantes nesse estudo são os coeficientes angulares e lineares das retas. Considerando que se pretende comprovar que são obtidas recuperações numericamente iguais às concentrações de fortificação, o coeficiente linear deve ser o mais próximo possível de zero e o coeficiente angular igual a 1. As retas construídas a partir do estudo de POLESE et al. (2002) (Figuras 4 e 5), mostraram excelentes coeficientes de correlação. As equações dessas retas são apresentadas na Tabela 6 .

\section{FIGURA 4 - LINEARIDADE DA RELAÇÃO ENTRE A CONCENTRAÇÃO RECUPERADA E A CONCENTRAÇÃO DE FORTIFICAÇÃO, COM NÍVEIS VARIANDO ENTRE 0,1 E $2,0 \mathrm{mg} / \mathrm{kg}$}

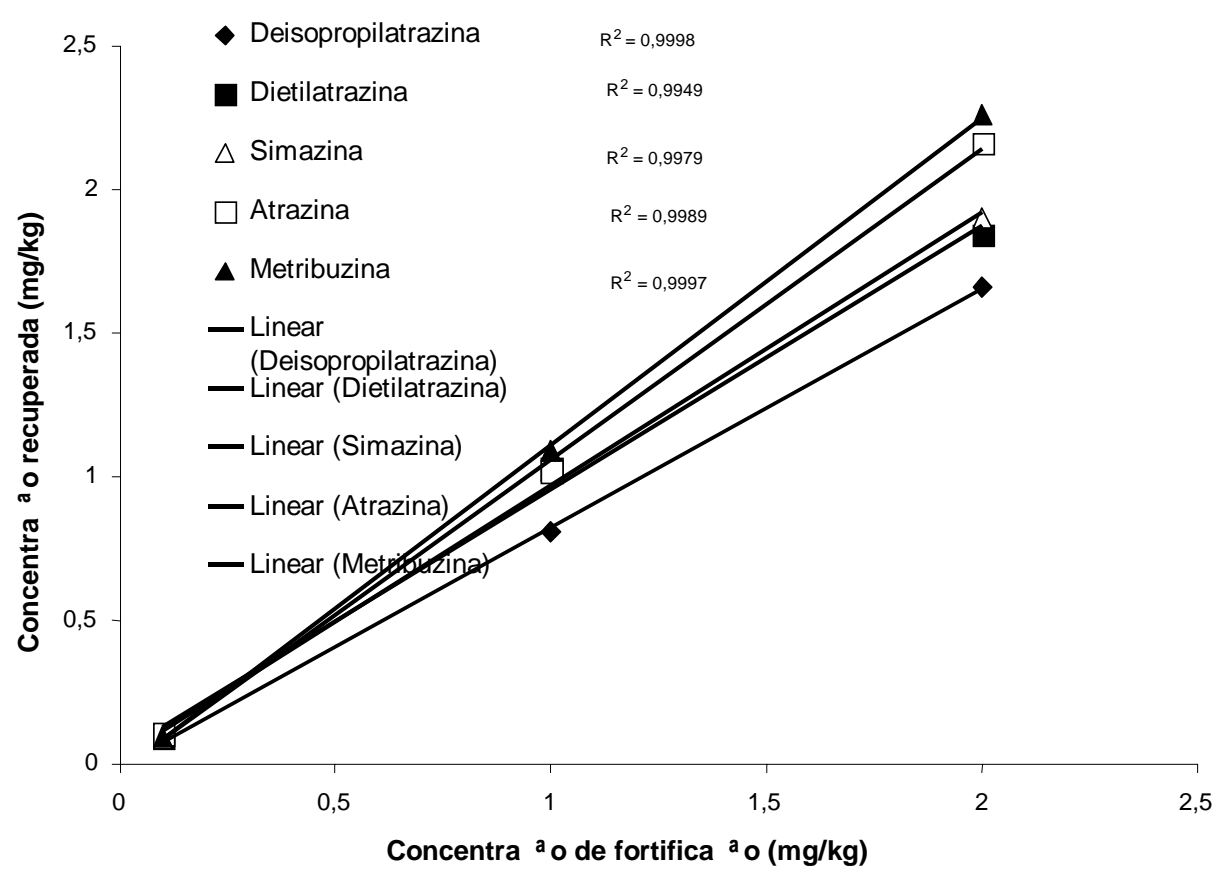



CONCENTRAÇÃO DE FORTIFICAÇÃO, COM NÍVEIS VARIANDO ENTRE 0,2 E $4,0 \mathrm{mg} / \mathrm{kg}$

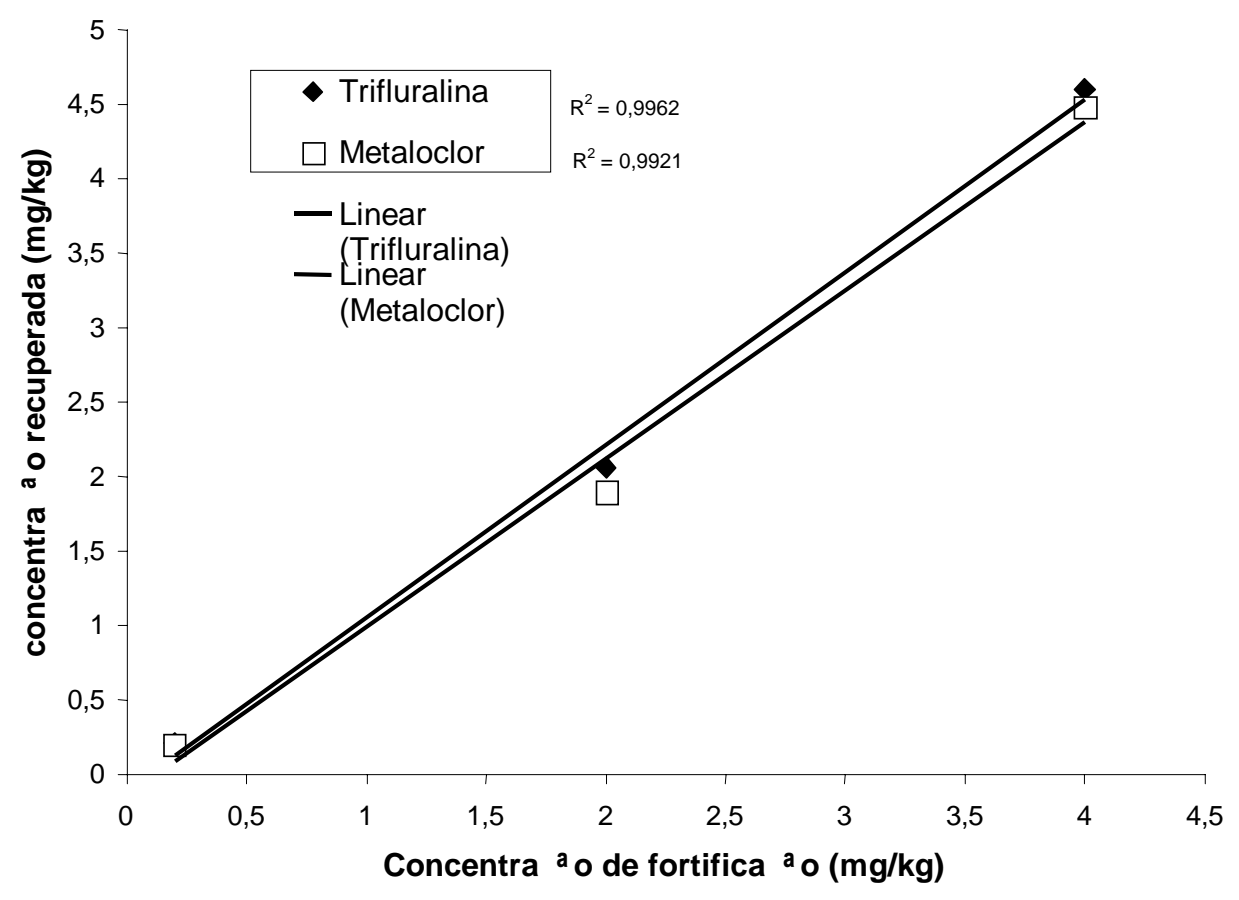

A Simazina e a Atrazina apresentaram os coeficientes angulares mais próximos da unidade e a Diisopropilatrazina o coeficiente linear mais próximo de zero. Como o trabalho estudado não forneceu dados suficientes para o teste $t$ dos valores de "a" e "b" das equações da reta não foi possível afirmar a existência de diferenças significativas entre esses valores, a nulidade e a unidade, respectivamente.

TABELA 6 - EQUAÇÕES DAS REGRESSÕES LINEARES PARA A RELAÇÃO ENTRE AS CONCENTRAÇÕES DE FORTIFICAÇÃO E AS ADICIONADAS

\begin{tabular}{l|c}
\hline Princ pio ativo & Equa ${ }^{\mathrm{a}} \mathbf{0}$ \\
\hline Diisopropilatrazina & $y=0,8299 x-0,0062$ \\
Dietilatrazina & $y=0,9164 x+0,0414$ \\
Simazina & $y=0,9497 x+0,0229$ \\
Atrazina & $y=1,0827 x-0,0237$ \\
Metribuzina & $y=1,1385 x-0,0271$ \\
Trifluralina & $y=1,1553 x-0,095$ \\
Metaloclor & $y=1,1273 x-0,1337$ \\
\hline
\end{tabular}




\subsection{LIMITES DE QUANTIFICAÇÃO E DE DETECÇÃO}

De acordo com o método de THIER e ZEUMER (1987), o Limite de Detecção (LD) é calculado com base nos resultados para o menor nível de fortificação. O Limite de Quantificação é o menor nível de concentração em que se conhecem a exatidão e a precisão do método $(26,36)$. Os valores de LD e LQ estão apresentados na Tabela 7.

\section{TABELA 7 - VALORES DE LD E LQ PARA OS SETE COMPOSTOS INVESTIGADOS}

\begin{tabular}{|c|c|c|}
\hline Pesticidas & LD $\left(m g k g^{-1}\right)$ & $L Q(m g k g-1)$ \\
\hline Diisopropilatrazina & 0,03 & 0,1 \\
\hline Dietilatrazina & 0,02 & 0,1 \\
\hline Trifluralina & 0,06 & 0,2 \\
\hline Simazina & 0,02 & 0,1 \\
\hline Atrazina & 0,02 & 0,1 \\
\hline Metribuzina & 0,04 & 0,1 \\
\hline Metolaclor & 0,01 & 0,2 \\
\hline
\end{tabular}

Fonte: POLESE et al., 2002 (35).

Os valores reportados foram considerados satisfatórios (suficientemente baixos), principalmente, para o propósito do método (a análise de resíduos de herbicidas em amostras de solo). Cabe ressaltar que o procedimento de THIER e ZEUMER é indicado para a determinação do limite de detecção por considerar não apenas a capacidade do equipamento em detectar a quantidade de composto que é injetada, mas todas as etapas do processo analítico, desde a extração até a detecção.

\section{CONCLUSÃO}

A validação é essencial para definir se métodos desenvolvidos estão completamente adequados aos objetivos a que se destinam, a fim de se obter resultados confiáveis que possam ser satisfatoriamente interpretados. Desta forma, possibilita o conhecimento das limitações e da confiabilidade nas medidas realizadas nas análises.

Dependendo do propósito do método, alguns dos parâmetros apresentados podem deixar de ser avaliados. A exatidão e a precisão do método constituem parâmetros sempre estudados, independente do seu propósito, exceto para métodos com objetivo apenas qualitativo.

Deve-se ressaltar que o método pode ser considerado validado, mesmo que alguns parâmetros não se enquadrem nos limites estabelecidos na literatura, mas que sejam criteriosamente conhecidos e, portanto, adequados aos objetivos do estudo a ser realizado.

\section{ABSTRACT}

ANALYTICAL METHODS VALIDATION: STRATEGY AND DISCUSSION

This paper presents the definitions of the parameters (selectivity, limits of detection and quantification, accuracy, precision, linearity, analytical graphic, sensitivity and ruggedness) considered on analytical methods validation procedures. The 
strategy to be adopted for determinations of these parameters depends on the purpose and the nature of the method. Some examples are presented for parameters evaluation in a validation procedure.

KEY-WORDS: ANALYTICAL METHODS-VALIDATION; VALIDATION CRITERIA.

\section{REFERÊNCIAS}

1 WALSH, M. C. Moving from official to traceable methods. Trends in Analytical Chemistry, v.18, p.616-623, 1999.

2 HUBERT, P. et al. The SFSTP guide on the validation of chromatographic methods for drug bioanalysis: from the Washington Conference to the laboratory. Anal. Chim. Acta, v. 391, p.135-139, 1999.

3 CAUSON, R. Validation of chromatographic methods in biomedical analysis: viewpoint and discussion. J. Chromatogr. B, v. 689, 175-180, 1997.

4 WIELING, J.; HENDRINKS, C.; TAMMINGA, W. J.; HEMPENIUS, J.; MENSINK, C. K.; OOSTERHUIS, B.; JONKMAN, J. H. G. Rational experimental desing for bioanalytical methods validation: illustration using an assay method for total captropil in plasma. J. Chromatogr. A, v. 730, p.381-394, 1996.

5 BRESSOLLE, F.; BROMET-PETIT, M.; AUDRAN, M. Validation of liquid chromatographic and gas chromatographic methods: applications to pharmacokinetics. J. Chromatogr. B, v.686, p.3-10, 1996.

6 CHASIN, A. M.; CHASIN, M.; SALVADORI, M. C. Validação de métodos cromatográficos em análises toxicológicas. R. Farm. Bioquim., v. 30, n. 2, p. 49-53, 1994.

7 FRANCOTTE, E.; DAVATZ, A.; RICHERT, P. Development and validation of chiral high-performance liquid chromatographic methods for the quantitation of valsartan and of the tosylate of valinebenzyl ester. J. Chromatogr. B, v. 686, p. 77-80, 1996.

8 McDONALD, R. D. The role of laboratory information management systems (LIMS) in analytical method validation. Anal. Chim. Acta, v. 391, p. 149-158 1999.

9 CURRIE, L. A.; SVEHLA, G. Nomenclature for the presentation of results of chemical analysis. Pure \& Appl. Chem., v. 66, n. 3, p. 595-908, 1994.

10 PEREIRA, D. M. C.; ARCOS, M. A. S. V.; AMARANTE Jr., O. P. de; CALDAS, E. P. A. Comparação de métodos dicromatométricos para determinação de ferro total em minérios de ferro. Anais Assoc. Bras. Quím., v. 49, n. 4, p. 198-203, 2000.

11 FEINBERG, M.; RAGUÈNÈS, N. Development and application of a standardized validation procedure for food chemistry laboratories. Anal. Chim. Acta., v. 391, p. 239-252, 1999.

12 VALIDATION of analytical methods: definitions and terminology. London: ICH, 1995. 5 p. (ICH Harmonised Tripartite Guideline). (CPMP/ICH/381/95).

13 BRITO, N. M. Resíduos de pesticidas organoclorados (OC) e organofosforados (OF) em matriz de coco: metodologia e aplicação. São Paulo, 2001. Dissertação (Mestrado em Química Analítica), Universidade Estadual Paulista.

14 VALIDATION of analytical procedures: metodology : ICH harmonised tripartite guideline. In: INTERNATIONAL CONFERENCE ON HARMONATION OF TECHINICAL REQUERIMENTS FOR REGISTRATION OF PHARMACEUTICALS FOR HUMAN USE, 1996. $8 \mathrm{p}$.

15 VALIDATION of analytical procedures: metodology. London: ICH, 1996. 9 p. (ICH Harmonised Tripartite Guideline). $\mathrm{CPMP} / \mathrm{ICH} / 281 / 95$

16 AMARANTE Jr., O. P. de; CALDAS, E. P. A.; BRITO, N. M.; SANTOS, T. C. R. dos; VALE, M. L. B. F. Validação de métodos analíticos: uma breve revisão. Cad. Pesq., v. 12, p. 116-131, 2001.

17 STUBBERUD, K. P.; ASTROM, O. Separation of ibuprofen, codeine phosphate, their degradation products and impurities by capillary electrophoresis: II Validation. J. Chromatogr. A., v. 826, p. 95-102, 1998.

18 CURRIE, L.A. Detection and quantification limits: origins and historical overview. Anal. Chim. Acta, v. 391, p. 127134, 1999.

19 CURRIE, L. A. Nomenclature in evaluation of analytical methods including detection and quantification capabilites (IUPAC Recommendations 1995). Anal. Chim. Acta., v. 391, p. 105-126, 1999.

20 GONZÁLEZ, A. G.; HERRADOR, M. A.; ASUERO, A. G. Intra-laboratory testing of method accuracy from recovery assays. Talanta, v. 48, p. 729-736, 1999. 

990, 1991.

ISO. International Standards Organization. ISO 5725: accuracy (trueness and precision) of measurement methods and results. Géneve, 1994. Part 1-4, 6.

VAN DER VOET, H.; VANRHIJIN, H.; VAN DER WIEL, H. J. Inter-laboratory, time, and fitness-for-purpose aspects of effective validation. Anal. Chim. Acta., v. 391, p. 159-171, 1999.

24 MAROTO, A.; RIU, J.; BOQUÉ, R.; RIUS, F. X. Estimating uncertainties of analytical results using informations from the validation process. Anal. Chim. Acta., v. 391, p. 173-185, 1999.

AMARANTE Jr., O. P. de. Determinação do ácido 2,4-diclorofenoxiacético e seu principal produto de degradação em solos de campos de cultivo de eucaliptos. São Luís, 2002. Dissertação (Mestrado), Universidade Federal do Maranhão.

THIER, H. P.; ZEUMER, H. Manual of pesticide analysis. New York: Verlag Chemie, 1987, p. 37-41.

WOOD, R. How to validate analytical methods. Trends Anal. Chem., v. 18, p. 624-632, 1999.

ANALYTICAL methods committee (Royal Society of Chemistry). Analyst, v.112, p 199-204, 1987.

CURRIE, L.; HORWITZ, W. IUPAC recommendations for defining and measuring detection and quantification limits. Analysis Magazine, v. 22, n. 5, p. M24-M26, 1994.

LONG, G. L.; WINEFORDNER, J. D. Limit of detection: a closer look at the IUPAC definition. Anal. Chem., v. 55, n. 7, p. 712A-724A, 1983.

31 HUBER, L. Validation of analytical methods: review and strategy. LC/GC International, Feb 1998, p. 96-105. Disponível em: <http://www.labcompliace. com/index.htm>. Acesso em 12 de jan. 2000.

32 NIJHUIS, A.; KNAAP, H. C. M. Van der; JONG, S. de; VANDEGINSTE, B. G. M. Strategy for ruggedness tests in chromatographic method validation. Anal. Chim. Acta, v. 391, p. 187-202, 1999.

VAN DER HEYDEN, Y.; JIMIDAR, M.; HUND, E.; NIEMEIJER, N.; PEETERS, R.; SMEYERS-VERBEKE, J.; MASSART, D. L.; HOOGMARTENS, J. Determination of system suitability limits with a robustness test. J. Chromatogr. A., v. 845, p. $145-154,1999$

34 ZOONEN, P. V.; HOOGERBRUGGE, R.; GORT, S. M.; WIEL, H. J. VAN DER; KLOOSTER, H. A. Some practical examples of method validation in the analytical laboratory. Trends Anal. Chem., v. 18, p. 584-593, 1999.

35 POLESE, L.; DORES, E. F. G. de C.; JARDIM, E. F. G.; NAVICKIENE, S.; RIBEIRO, M. L. Determination of herbicides in soil samples. Eclét. Quím., v. 27, p. 249-254, 2002.

36 BRITO, N. M.; AMARANTE JUNIOR, O. P. de; POLESE, L.; SANTOS, T. C. R. dos; RIBEIRO, M. L. Avaliação da exatidão e da precisão de métodos de análise de resíduos de pesticidas mediante ensaios de recuperação. Pesticidas: $R$. Ecotoxicol. Meio Ambiente, v. 12, p. 155-168, 2002. 